\title{
THE HODGE THEORY OF FLAT VECTOR BUNDLES ON A COMPLEX TORUS
}

BY

\author{
JEROME WILLIAM HOFFMAN ${ }^{1}$
}

\begin{abstract}
We study the Hodge spectral sequence of a local system on a compact, complex torus by means of the theory of harmonic integrals. It is shown that, in some cases, Baker's theorems concerning linear forms in the logarithms of algebraic numbers may be applied to obtain vanishing theorems in cohomology. This is applied to the study of Betti and Hodge numbers of compact analytic threefolds which are analogues of hyperelliptic surfaces. Among other things, it is shown that, in contrast to the two-dimensional case, some of these varieties are nonalgebraic.
\end{abstract}

\section{Introduction.}

(0.0) The purpose of this paper is to determine the Hodge spectral sequence associated with a vector bundle with integrable connection on a complex torus. Such vector bundles typically arise as the hypercohomology sheaves attached to a proper and smooth morphism $f: Y \rightarrow X$ where $X$ is a complex torus. Consequently our results will have application to the study of those manifolds which admit such fiberings over tori.

$(0.1)$ In outline, the main result is as follows: Let $(\mathscr{W}, \nabla)$ be a holomorphic vector bundle with integrable connection on a complex torus $X=V / L$, where $L \subset V$ is a lattice in a complex vector space. Let $W=\mathscr{W} \nabla$ be the corresponding C-local system. We compute $H^{q}\left(X, \Omega^{p}(\mathcal{W})\right)$ and $\bar{H}^{k}(X, \underline{W})$ in terms of harmonic differential forms via Hodge's theorem.

First we show that W admits a global real analytic frame. Relative to this frame, $C^{\infty}$ differential forms in $\mathscr{W}$ may be represented by $C^{\infty} m$-vectors of differential forms $\varphi$ on $V$ which are $L$-periodic ( $m=$ rank $\mathscr{W}$ ). Using the frame, a Hermitian metric may be introduced into the fibers of $\mathcal{W}$ in such a way that the harmonic theory takes on a simple form. Specifically, the Laplace equations $\Delta \varphi=0$ become, upon Fourier transform, $\Delta(\underline{m}) \hat{\varphi}(\underline{m})=0, \underline{m} \in \mathbf{Z}^{2 n}(n=\operatorname{dim} V)$. Each of these is a finite-dimensional matrix equation. We prove (for the choices made):

(a) For $H^{k}(X, \underline{W}), \Delta(\underline{m}) \hat{\varphi}(\underline{m})=0$ has only the zero solution if $\underline{m} \neq 0$. Hence $H^{k}(X, \underline{W}) \simeq \operatorname{ker} \Delta(0)$.

(b) For $H^{q}\left(X, \Omega^{p}(\mathcal{Q})\right)$, only a finite number of $\underline{m}$ can occur for which det $\Delta(\underline{m})$ $=0$ and these are identified by a diophantine condition involving the logarithms of the periods of $X$. These $\underline{m}$ are "singular". Therefore, modulo determining the singular $\underline{m}$, all computations are reduced to a finite amount of linear algebra.

Received by the editors October 28, 1980.

1980 Mathematics Subject Classification. Primary 14C30; Secondary 32J25, 14K20.

'Partially supported by NSF Grant MCS-8002231. 
(0.2) The diophantine problem mentioned in (b) above is exactly the same one that Baker has studied in his papers on the linear forms in the logarithms of algebraic numbers. By applying his theorems, we can obtain some vanishing theorems for the cohomologies of local systems on complex tori.

(0.3) In the last section, we study some threefolds which are analogues of the hyperelliptic surfaces. In contrast with the 2-dimensional case, nonalgebraic and even non-Kaehlerian ones can arise. One challenging problem is to try and use these methods to obtain more examples of non-Kaehlerian deformations of Kaehler manifolds.

(0.4) This paper is the essential content of my Ph.D. thesis. I would like to thank my advisor Heisuke Hironaka as well as Philip Griffiths and David Mumford for help and encouragement while this work was in preparation.

\section{Preliminaries.}

(1.0) Let $V$ be a $\mathrm{C}$-vector space of dimension $n$, and $L \subset V$ a lattice so that $X=V / L$ is a compact complex torus. We let $z_{1}, \ldots, z_{n}$ be coordinates on $V$. $z_{j}=x_{j}+i y_{j}$ with $i=\sqrt{-1}$. We let $e_{1}, \ldots, e_{2 n}$ be a $\mathbf{Z}$-basis of $L$. Thus, any $v \in V$ may be written uniquely in the form $v=\sum_{j=1}^{2 n} v_{j} e_{j}$ with $v_{j} \in \mathbf{R}$.

We have $e_{k}=\left(\varepsilon_{i k}, \ldots, \varepsilon_{n k}\right)=\left(\varepsilon_{j k}\right)=\left(\tau_{j k}\right)+i\left(\nu_{j k}\right)$ where $\tau_{j k}, \nu_{j k} \in \mathbf{R}$. Hence,

$$
v=\left(\sum_{k=1}^{2 n} \tau_{j k} v_{k}\right)+i\left(\sum_{k=1}^{2 n} \nu_{j k} v_{k}\right) .
$$

Or, in matrix form,

$$
\left(\begin{array}{l}
\tau \\
\nu
\end{array}\right)(v)=\left(\begin{array}{l}
x \\
y
\end{array}\right)
$$

Set $(t, u)=\left(\begin{array}{l}\tau \\ \nu\end{array}\right)^{-1} ;$ then

$$
v_{j}=\sum_{k=1}^{n}\left(t_{j k} x_{k}+u_{j k} y_{k}\right)
$$

Define

$$
\partial_{a}=\partial / \partial z_{a}, \quad \bar{\partial}_{b}=\partial / \bar{\partial}_{b}, \quad d_{j}=\partial / \partial v_{j} .
$$

Then if $\lambda_{j k}=\frac{1}{2}\left(t_{j k}+i u_{j k}\right)$ we get

$$
\partial_{a}=\sum_{j=1}^{2 n} \bar{\lambda}_{j a} d_{j} \quad \text { and } \quad \bar{\partial}_{b}=\sum_{j=1}^{2 n} \lambda_{j b} d_{j} .
$$

We have

$$
\operatorname{det}(\lambda, \bar{\lambda}) \neq 0 \text {. }
$$

(1.1) We follow the usual conventions on multi-indices for differential forms, cf. [9]. Generally, $I, J$, etc. $\subseteq\{1, \ldots, 2 n\}$ and $A, B \subseteq\{1, \ldots, n\}$.

2. Vector bundles on complex tori.

(2.0) A holomorphic rank $m$ vector bundle $\mathscr{W}$ on $X$ is given by a collection of analytic $\mathrm{GL}_{m}(\mathbf{C})$-valued functions on $V\left\{\rho_{\lambda}(v) \mid \lambda \in L\right\}$ satisfying the cocycle 
$\rho_{\lambda+\lambda^{\prime}}(v)=\rho_{\lambda}\left(v+\lambda^{\prime}\right) \rho_{\lambda^{\prime}}(v)$ to all pairs $\lambda, \lambda^{\prime} \in L$. Cohomologous cocycles give isomorphic bundles and conversely.

Let $\mathscr{W}$ be defined by a cocycle $\rho_{\lambda}$. Let $A^{p, q}(\mathcal{W})$ denote the space of global $C^{\infty}$ W-valued $(p, q)$-forms on $X$. An element $\varphi \in A^{p, q}(\mathcal{W})$ is the same thing as a $C^{\infty}$ $\mathbf{C}^{m}$-valued $(p, q)$-form on $V$ such that

$$
\begin{gathered}
\varphi(v+\lambda)=\rho_{\lambda}(v) \varphi(v) \quad \text { for all } \lambda \in L . \\
A^{k}(\mho) \underset{\text { def }}{=} \underset{p+q=k}{\oplus} A^{p, q}(\mho) .
\end{gathered}
$$

(2.1) Let $\nabla: \mathscr{W} \rightarrow \Omega_{X}^{1} \otimes_{\hat{O}_{x}} \mathcal{W}=\Omega^{1}(\mathscr{W})$ be a flat (= integrable) holomorphic connection. Let $\underline{W}=\mathcal{Q} \int^{\nabla}$ be the sheaf kernel of $\nabla$ whose sections are called horizontal holomorphic sections of $\mathcal{W}$. This is a C-local system on $X$ of $\operatorname{rank}=\mathrm{rk}(\mathcal{W})$ by Frobenius' theorem. It is known that we may choose a cocycle for 015 so that (a) $\rho_{\lambda}(v)$ is independent of $v$, and (b) the connection matrices of $\nabla$ relative to $\rho_{\lambda}$ are identically 0 . Condition (a) means that $\lambda \mapsto \rho_{\lambda}$ is an $m$-dimensional representation of the abelian group $L$, and condition (b) means that, with the identification (2.0.0), $\nabla \varphi=d \varphi$ where $d$ is the usual exterior differentiation on each entry of the $m$-vector of differential forms $\varphi$. This understood, we sometimes write $d$ for $\nabla$.

The representation $\rho_{\lambda}$ attached to $(\mathcal{W}, \nabla)$ is called the monodromy representation. Conversely, given a representation $\rho: L \rightarrow \mathrm{GL}(W)$ where $W$ is an $m$-dimensional C-vector space, we obtain a local system $\underline{W}_{\rho}$ on $X$, and a vector bundle with flat connection $\left(\vartheta_{\rho}=\underline{W}_{\rho} \otimes_{\mathrm{C}} \vartheta_{X}, \nabla=1 \otimes d\right)$. We denote by $W_{\rho}$ the vector space $W$ viewed as $L$-module via $\rho$. We set $\rho_{\lambda}=\rho(\lambda)$.

Because $X$ is a $K(\pi, 1)$ with $\pi=L$, we get

(2.1.0) Proposition. There is a canonical isomorphism $H^{k}\left(X, \underline{W}_{\rho}\right) \simeq H^{k}\left(L, W_{\rho}\right)$ where the right-hand side is group cohomology.

It follows that $H^{k}\left(X, \underline{W}_{\rho}\right)$ is independent of the complex structure on $X$ (i.e. the position of $L$ in $V$ ).

(2.2) Let $\mho_{\rho}=(\mathscr{W}, \nabla)$ be a flat vector bundle on $X$. We denote by $\Omega^{*}\left(\mathcal{W}_{\rho}\right)=$ $\Omega^{*}(U, \nabla)$ the de Rham complex (augmented) $0 \rightarrow \underline{W}_{\rho} \stackrel{i}{\rightarrow} \mathcal{W}_{\rho} \stackrel{\nabla}{\rightarrow} \Omega^{1}\left(\mathcal{W}_{\rho}\right) \stackrel{\nabla}{\rightarrow}$ $\Omega^{2}\left(\mathcal{W}_{\rho}\right) \rightarrow \cdots$.

(2.2.0) THEOREM. There is a canonical isomorphism $H^{k}\left(X, \underline{W}_{\rho}\right) \sim \mathbf{H}^{p+q}\left(X, \Omega^{*}\left(\mathscr{W}_{\rho}\right)\right)$ and consequently a spectral sequence $E_{1}^{p, q}=H^{q}\left(X, \Omega^{p}\left(\mathcal{Q V} \int_{\rho}\right)\right) \Rightarrow H^{p+q}\left(X, \underline{W}_{\rho}\right)$ abutting to a filtration on the limit (Hodge filtration).

It is well known that, as a consequence of (2.2.0), one has

$$
\begin{cases}H^{k}\left(X, \underline{W}_{\rho}\right)=H^{k}\left(A^{*}\left(\mho_{\rho}\right), d\right) & \text { (de Rham), } \\ H^{q}\left(X, \Omega^{p}\left(\mathscr{U}_{\rho}\right)\right)=H^{q}\left(A^{p, *}\left(\mathscr{\mho}_{\rho}\right), \bar{\partial}\right) & \text { (Dolbeault). }\end{cases}
$$

Furthermore, the differential in the spectral sequence is $\partial$ where $d=\partial+\bar{\partial}$. 
(2.3) We have $W_{\rho}=\bigoplus_{\chi} W_{\rho}(\chi)$ where $W_{\rho}(\chi) \subset W$ is the subvector space on which $\rho$ acts via matrices of the form

$$
\left(\begin{array}{ccc}
\chi(\lambda) & & * \\
& \ddots & \\
0 & & \chi(\lambda)
\end{array}\right)
$$

with $\chi: L \rightarrow \mathrm{C}^{*}$ a homomorphism. $W_{\rho}(\chi)$ or $W(\chi)$ is called the isotypic component with diagonal character $\chi$. We obtain a decomposition of the local system $\underline{W}_{\rho}$ and hence of the spectral sequence according to the $\chi$. It is sufficient to study the Hodge theory of isotypic local systems.

(2.4) Let $\rho_{j}=\rho\left(e_{j}\right)$ with $\rho: L \rightarrow \operatorname{GL}(W)$ a representation. Note that $\left[\rho_{j}, \rho_{k}\right]=0$ for all $j, k([]=$, commutator $)$.

(2.4.0) Lemma. There is a unique set of matrices $R_{j}$ such that

(a) $\exp R_{j}=\rho_{j}, R_{j}$ upper triangular if $\rho_{j}$ is,

(b) $\left[R_{j}, R_{k}\right]=0$ for all $j, k$,

(c) $0 \leqslant \operatorname{Im} \sigma\left(R_{j}\right)<2 \pi$ where $\sigma(R)=$ spectrum of $R$.

It follows from (c) that

(d) $\operatorname{det}\left(2 \pi i m+R_{j}\right) \neq 0$ if $m \in \mathbf{Z}-\{0\}$.

In view of (2.3) it is clear that $R_{j}$ may be chosen to be decomposed into block form according to the isotypic components, and by (2.4.0)(a) each block taken in upper triangular form (since we may simultaneously render any commuting family of matrices in that form).

Now choose $R_{j}$ as above and define, for $v=\Sigma v_{j} e_{j}, \rho(v)=\exp \left(\sum v_{j} R_{j}\right)$. Then, because $\left[R_{j}, R_{k}\right]=0$ for all $j, k$, we have $\rho\left(v+v^{\prime}\right)=\rho(v) \rho\left(v^{\prime}\right)$. In particular $\rho(v+\lambda)=\rho(\lambda) \cdot \rho(v)$ for all $\lambda \in L$. Let $r_{\mu}(v)$ denote the $\mu$ th column vector of $\rho(v)$. Clearly $r_{\mu}(v+\lambda)=\rho(\lambda) r_{\mu}(v)$. Because of (2.0.0) this means that $r_{\mu}(v)$ is a real analytic global section of $\mathscr{U}_{\rho}$. Since $\operatorname{det} \rho(v) \neq 0$ we have proved

(2.4.1) Proposition. The $r_{\mu}(v)$ for $\mu=1, \ldots, m$ constitute a global real-analytic frame for the bundle of $_{\rho}$.

Note that the $r_{\mu}(v)$ are not in general horizontal. We write, for $\varphi \in A^{p, q}\left(\mathcal{Q} \int_{\rho}\right)$,

$$
\left\{\begin{array}{l}
\varphi=\sum_{A, B} \varphi_{A_{p} \bar{B}_{q}}(v) d z_{A_{p}} \wedge d \bar{z}_{B_{q}}, \quad \varphi_{A_{p} \bar{B}_{q}}(v+\lambda)=\rho(\lambda) \varphi_{A_{p} \bar{B}_{q}}(v), \\
\varphi_{A_{p} \bar{B}_{q}}(v)=\sum_{\mu=1}^{m} \varphi_{A_{p} B_{q}(v) r_{\mu}(v)} \\
\quad \text { where } \varphi_{A_{p} \bar{B}_{q}}^{\mu}(v+\lambda)=\varphi_{A_{p} B_{q}}^{\mu}(v) \text { is } C^{\infty} L \text {-periodic. }
\end{array}\right.
$$

We have a Fourier decomposition

$$
\left\{\begin{array}{l}
\varphi=\sum_{\underline{m}} \varphi(\underline{m}), \quad \underline{m}=\left(m_{1}, \ldots, m_{2 n}\right) \in \mathbf{Z}^{2 n}, \\
\varphi(\underline{m})=\sum_{A, B}\left(\sum_{\mu} \hat{\varphi}_{A_{p} B_{q}}^{\mu}(\underline{m}) e(\underline{m} \cdot v) r_{\mu}(v)\right) d z_{A_{p}} \wedge d \bar{z}_{B_{q}}
\end{array}\right.
$$


where denotes Fourier transform of the $C^{\infty} L$-periodic function $f$,

$$
\left\{\begin{array}{l}
f(v)=\sum_{\underline{m}} \hat{f}(\underline{m}) e(\underline{m} \cdot v), \quad \underline{m} \cdot v=\sum_{j=1}^{2 n} m_{j} v_{j}, \\
\hat{f}(\underline{m})=\int_{X} f(v) e(-\underline{m} \cdot v) d v \quad \text { where } e(z)=\exp (2 \pi i z), \\
\int_{X} d v=1, \quad d v \text { is Haar measure } d v_{1} \wedge \cdots \wedge d v_{2 n} .
\end{array}\right.
$$

We set

$$
\left\{\begin{array}{l}
\tilde{\varphi}_{A_{p}} \bar{B}_{q}(v)=\left(\varphi_{A_{p}}^{1} \bar{B}_{q}(v), \ldots, \varphi_{A_{p} B_{q}}^{m}-(v)\right), \\
\left.\hat{\varphi}(\underline{m})=\left(\hat{\varphi}_{A_{p}}^{1} \bar{B}_{q}(\underline{m}), \ldots, \hat{\varphi}_{A_{p} B_{q}}^{m}-\underline{m}\right)\right)
\end{array}\right.
$$

as column vectors.

Defining

$$
\left\{\begin{aligned}
\tilde{\varphi}(v)= & \sum_{A, B} \tilde{\varphi}_{A_{p} \bar{B}_{q}}(v) d z_{A_{p}} \wedge d \bar{z}_{B_{q}} \\
& \in C^{\infty}(X) \otimes_{\mathbf{C}}\left(W \otimes_{\mathbf{C}} \wedge{ }^{p, q} V\right), \\
\hat{\varphi}(\underline{m})= & \sum_{A, B} \hat{\varphi}_{A_{p} \bar{B}_{q}}(\underline{m}) d z_{A_{p}} \wedge d \bar{z}_{B_{q}} \in W \otimes_{\mathbf{C}} \wedge{ }^{p, q} V
\end{aligned}\right.
$$

gives

$$
\varphi(v)=\rho(v) \tilde{\varphi}(v)=\rho(v) \sum_{\underline{m}} \hat{\varphi}(\underline{m}) e(m \cdot v) .
$$

We have similar formulae and notation for $\varphi \in A^{k}\left(\mathcal{Q} \int_{\rho}\right)$ written as $\varphi=$ $\Sigma_{I} \varphi_{I_{k}}(v) d v_{I_{k}}$.

(2.5) Since $\partial \rho(v) / \partial v_{k}=d_{k} \rho(v)=R_{k} \rho(v)=\rho(v) R_{k}$, we get

$$
d_{k} r_{j}(v)=\sum_{l=1}^{m}\left(R_{k}\right)_{l j} r_{l}(v) \text {. }
$$

Or

$$
\left(d_{k} \varphi\right) \tilde{\varphi}=\left(d_{k}+R_{k}\right) \tilde{\varphi}=D_{k} \tilde{\varphi} \quad \text { for } \varphi \in A^{\circ}\left(\mathcal{Q}_{\rho}\right) .
$$

Also

$$
\left\{\begin{array}{l}
\left(\partial_{a} \varphi\right) \tilde{\varphi}=\left(\sum_{j} \bar{\lambda}_{j a} D_{j}\right) \tilde{\varphi}=D_{a}^{\prime} \tilde{\varphi} \\
\left(\bar{\partial}_{b} \varphi\right) \tilde{\varphi}=\left(\sum_{j} \lambda_{j b} D_{j}\right) \tilde{\varphi}=D_{b}^{\prime \prime} \tilde{\varphi}
\end{array}\right.
$$

Taking Fourier transforms yields

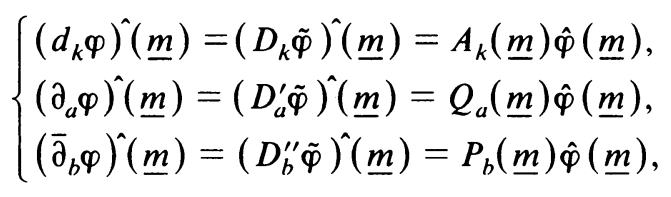


where

$$
\left\{\begin{array}{l}
A_{k}(\underline{m})=2 \pi i m_{k}+R_{k}, \quad Q_{a}(\underline{m})=\sum_{j} \bar{\lambda}_{j a} A_{j}(\underline{m}), \\
P_{b}(\underline{m})=\sum_{j} \lambda_{j b} A_{j}(m) .
\end{array}\right.
$$

We deduce from all this:

$$
\left\{\begin{array}{l}
\text { For } \varphi=\sum_{I} \varphi_{I_{k}}(v) d v_{I_{k}} \in A^{k}\left(\mathcal{U}_{\rho}\right), \\
\nabla \varphi=d \varphi=\rho(v) \sum_{\underline{m}}\left(\sum_{I, j} A_{j}(\underline{m}) \hat{\varphi}_{I_{k}}(\underline{m}) d v_{j} \wedge d v_{I_{k}}\right) e(\underline{m} \cdot v) ; \\
\text { For } \varphi=\sum_{A, B} \varphi_{A_{p} \bar{B}_{q}}(v) d z_{A_{p}} \wedge d \bar{z}_{B_{q}} \in A^{p, q}\left(\vartheta \int_{\rho}\right), \\
\bar{\partial} \varphi=(-1)^{p} \rho(v) \sum_{\underline{m}}\left(\sum_{A, B, b} P_{b}(\underline{m}) \hat{\varphi}_{A_{p} \bar{B}_{q}}(\underline{m}) d z_{A_{p}} \wedge d \bar{z}_{b} \wedge d \bar{z}_{B_{q}}\right) e(\underline{m} \cdot v) .
\end{array}\right.
$$

Let $A^{*}\left(\mathcal{W}_{\rho}(\underline{m})\right)$ denote the complex $W \otimes \wedge^{*}\left(\mathbf{C}^{2 n}\right)$ with differential

$$
d(\underline{m})\left(w \otimes d v_{I_{k}}\right)=\sum_{j} A_{j}(\underline{m}) w \otimes d v_{j} \wedge d v_{I_{k}}
$$

and let $A^{p, *}\left(\mathcal{Q} \int_{\rho}(\underline{m})\right)$ denote the complex $W \otimes \wedge^{p, *}\left(\mathbf{C}^{n}\right)=\bigoplus_{A} W \otimes \wedge^{*}\left(\mathbf{C}^{n}\right) d z_{A}$ with differential

$$
\bar{\partial}(\underline{m})\left(w \otimes d z_{A_{p}} \wedge d \bar{z}_{B_{q}}\right)=(-1)^{p} \sum_{b} P_{b}(\underline{m}) w \otimes d z_{A_{p}} \wedge d \bar{z}_{b} \wedge d \bar{z}_{B_{q}}
$$

We see from (2.5.4) that $\varphi \rightarrow \oplus \hat{\varphi}(\underline{m})$ is an injection of complexes:

$$
\left\{\begin{aligned}
& f:\left(A^{*}\left(\mathcal{W}_{\rho}\right), d\right) \tilde{\hookrightarrow} \underset{\underline{m}}{\bigoplus}\left(A^{*}\left(\mathcal{U}_{\rho}(\underline{m})\right), d(\underline{m})\right), \\
& f_{1}:\left(A^{p, *}\left(\mathcal{W}_{\rho}\right), \bar{\partial}\right) \tilde{\hookrightarrow} \underset{\underline{m}}{\bigoplus}\left(A^{p, *}\left(\mathcal{U S}_{\rho}(\underline{m})\right), \bar{\partial}(\underline{m})\right) .
\end{aligned}\right.
$$

Recall,

(2.6.1) Definition. Let $T=\left(T_{1}, \ldots, T_{n}\right)$ be a commuting family of endomorphisms of a vector space $W$. The Koszul complex of $T$ is the complex $W \otimes \wedge^{*}\left(\mathbf{C}^{n}\right)$ with differential

$$
d\left(w \otimes e_{i_{1}} \wedge \cdots \wedge e_{i_{k}}\right)=\sum_{j} T_{j}(w) \otimes e_{j} \wedge e_{i_{1}} \wedge \cdots \wedge e_{i_{k}}
$$

Thus, $A^{P, *}\left(\mathscr{U}_{\rho}(\underline{m})\right)$ is the direct sum $\left(\begin{array}{c}n \\ p\end{array}\right)$ times the Koszul complex of $T=$ $\left(P_{1}(\underline{m}), \ldots, P_{n}(\underline{m})\right)$.

We shall see that the isomorphisms (2.6.0) are injections of elliptic complexes, once suitable metrics are chosen.

(2.7) Remark. Generally, we use the basis $d v_{I}$ rather than $d z_{A} \wedge d \bar{z}_{B}$ when dealing with the de Rham (rather than Dolbeault) complex. This is purely a matter of convenience.

(2.8) Noting that the differential in the spectral sequence (2.2.0) is that induced by $\partial$, which on Fourier components acts as $\Sigma Q_{a}(\underline{m}) d z_{a} \wedge$, we see that the entire Hodge 
spectral sequence (2.2.0) admits of a Fourier decomposition $E_{*} \simeq \bigoplus_{\underline{m}} E_{*}(\underline{m})$. This remark was made to me by Mumford. The key point in all this is that $\rho(v)$ and $e(m \cdot v)$ are eigenvectors of all constant coefficient differential operators on $V$ (i.e. $d_{k} \rho(v)=$ const $\left.\rho(v)\right)$.

(2.9) We put a metric in the fibers of $\mho_{\rho}$ as follows: For $\varphi, \psi \in A^{\circ}\left(\mho_{\rho}\right)$ we define their pointwise inner product as

$$
(\varphi(v), \psi(v))={ }^{t} \tilde{\varphi}(v) \cdot \bar{\psi}(v),
$$

remembering that $\varphi(v)=\rho(v) \tilde{\varphi}(v)$ with $\varphi$ periodic so that the above expression is a function on $X$ as it should be. This is also

$$
(\varphi(v), \psi(v))={ }^{t} \varphi(v){ }^{t} \rho(-v) \overline{\rho(-v)} \overline{\psi(v)}={ }^{t} \varphi(v) h(v) \overline{\psi(v)}
$$

where $h(v)$ is $C^{\infty}$ Hermitian, positive definite on $V$ so that

$$
{ }^{t} \rho(\lambda) h(v+\lambda) \overline{\rho(\lambda)}=h(v) .
$$

We let $k(v)={ }^{t} h(v)$. Note that the metric $h$ in the fibers of $\vartheta_{\rho}$ is just the pull back under the isomorphism (real-analytic) of $\mho_{\rho}$ with the trivial bundle of the usual Hermitian metric $\sum_{\mu=1}^{m} w_{\mu} \bar{w}_{\mu}$ in the trivial bundle.

We have a metric $d s^{2}=\Sigma d z_{\alpha} \otimes d \bar{z}_{\alpha}=\Sigma g_{i j} d v_{i} \otimes d v_{j}$ on $X$. Note that the $g_{i j}$ are constants. Let $\omega(v)$ denote the volume element of this metric. Note that $\omega(v)=c \cdot d v$ where $c$ is a constant and $d v$ is Haar measure.

Given $h$ and $d s^{2}$ the spaces $A^{p, q}\left(\mathscr{W}_{\rho}\right)$ have an inner product. In this case, the $d z_{A_{p}} \wedge d \bar{z}_{B_{q}}$ are orthogonal to one another in the metric $d s^{2}$ and of unit length. So the inner product on $A^{p, q}\left(\mathcal{W}_{\rho}\right)=\bigoplus_{A, B} A^{\circ}\left(\mathcal{W} \int_{\rho}\right) d z_{A} \wedge d \bar{z}_{B}$ is given once one knows what it is on $A^{\circ}\left(\mathcal{\vartheta}_{\rho}\right)$. For $\varphi, \psi \in A^{\circ}\left(\mathcal{U}_{\rho}\right)$ we have

$$
\begin{aligned}
(\varphi, \psi) & =\int_{X}(\varphi(v), \psi(v)) \omega(v)=c \cdot \int_{X}(\varphi(v), \psi(v)) d v \\
& =c \cdot \sum_{\mu=1}^{m} \int \tilde{\varphi}^{\mu}(v) \overline{\tilde{\psi}^{\mu}(v)} d v \\
& =c \cdot \sum_{\mu=1}^{m} \sum_{\underline{m}} \hat{\varphi}^{\mu}(\underline{m}) \overline{\hat{\psi}^{\mu}(\underline{m})} \quad \text { by Plancherel's theorem } \\
& =c \cdot \sum_{\underline{m}}(\hat{\varphi}(\underline{m}), \hat{\psi}(\underline{m})) .
\end{aligned}
$$

It is now clear that (up to a universal constant) the injections (2.6.0) are metric isomorphisms, where the spaces $A^{k}\left(\mathscr{\vartheta}_{\rho}(m)\right)=W \otimes_{\mathbf{C}} \wedge^{k}\left(\mathbf{C}^{2 n}\right)$ have that metric which comes from the metric $d s^{2}=\Sigma d z_{\alpha} \otimes d z_{\alpha}$ on $\mathbf{C}^{n}=\oplus \mathbf{C} d z_{\alpha}$ and the usual Hermitian inner product on the space $W=\mathbf{C}^{m}$.

(2.10) Once the spaces $A^{p, q}\left(\mathcal{U W}_{\rho}\right)$ have an inner product the operators $d, \bar{\partial}$ have adjoints $\delta$ and $\vartheta$, respectively, and corresponding Laplace-Beltrami operators $\nabla=$ $d \delta+\delta d$ and $\square=\bar{\partial} \vartheta+\vartheta \bar{\partial}$. Let

$$
\begin{aligned}
\mathcal{H}_{\Delta}^{k}\left(\mathscr{W}_{\rho}\right) & =\left\{\varphi \in A^{k}\left(\mathscr{W}_{\rho}\right) \mid \Delta \varphi=0\right\}, \\
\mathcal{H}_{\square}^{p, q}\left(\mathcal{W}_{\rho}\right) & =\left\{\varphi \in A^{p, q}\left(\mathscr{W}_{\rho}\right) \mid \square \varphi=0\right\} .
\end{aligned}
$$


Then Hodge's theorem asserts that there is an isomorphism

$$
H^{k}\left(X, \underline{W}_{\rho}\right) \simeq \mathcal{H}_{\Delta}^{k}\left(\mathscr{U}_{\rho}\right), \quad H^{q}\left(X, \Omega^{p}\left(\mathcal{Q U}_{\rho}\right)\right) \simeq \mathcal{H}_{\square}^{p, q}\left(\mathscr{U}_{\rho}\right) .
$$

We have

$$
\begin{cases}\vartheta \varphi=-k^{-1} * \partial(k * \varphi), & \varphi \in A^{p, q}\left(\mathcal{Q} \int_{\rho}\right), \\ \delta \varphi=-k^{-1} * d(k * \varphi), & \varphi \in A^{k}\left(\mathcal{Q} \int_{\rho}\right)\end{cases}
$$

$k={ }^{t} h . *$ is the usual $*$ operator on each component of $\varphi$.

(2.11) Because the injections (2.6.0) are metric isomorphisms, they are also isomorphisms as elliptic complexes. This means that $f \circ \delta \circ f^{-1}=\bigoplus \delta(\underline{m})$ and $f_{1} \circ \vartheta \circ f_{1}^{-1}=\bigoplus \vartheta(\underline{m})$ and similarly for the Laplacians. Here, $\delta(\underline{m})$ is the adjoint to $d(\underline{m})$ for the inner product in $A^{*}\left(\mathscr{Q} \int_{\rho}(\underline{m})\right)$ (cf. (2.9)). One could also verify this directly using (2.10.2). Letting $\mathcal{H}_{\Delta(m)}^{k}\left(\mathcal{O} \int_{\rho}(\underline{m})\right)$ denote $\left\{\varphi \in A^{k}\left(\mathcal{Q} \int_{\rho}(\underline{m})\right) \mid \Delta(\underline{m}) \varphi=0\right\}$ we then have isomorphisms

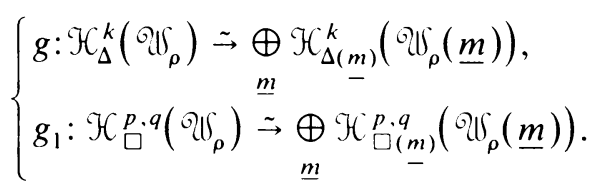

Similarly, the harmonic projection becomes isomorphic to $\bigoplus \operatorname{pr}(\underline{m})$ where $\operatorname{pr}(\underline{m})$ is the harmonic projection in $A^{k}\left(\mathscr{Q}_{\rho}(\underline{m})\right)$.

(2.12) We will never actually need it, but we include an explicit formula for $\square(\underline{m})$ :

If

$$
\varphi=\sum_{A, B} \varphi_{A, \bar{B}} d z_{A} \wedge d \bar{z}_{B} \in A^{p, q}\left(\mathscr{Q U}_{\rho}\right)(\underline{m})
$$

then

$$
(\square(\underline{m}) \varphi)_{A, B}=\sum_{h}\left(\left(P_{b}(\underline{m})^{*} P_{h}(\underline{m})\right) \varphi_{A, \bar{B}}-\sum_{\nu=1}^{q}\left[P_{b}(\underline{m})^{*}, P_{b_{\nu}}(\underline{m})\right] \varphi_{A, \bar{B}(\nu ; b)}\right),
$$

where $\bar{B}=\left(b_{1}, \ldots, b_{\nu}, \ldots, b_{q}\right), \bar{B}(\nu ; b)=\left(b_{1}, \ldots, b_{\nu-1}, b, b_{\nu+1}, \ldots, b_{q}\right)$, remembering that tensors are alternating in their index sets.

3. The theorems.

(3.0) $X$, Od $_{\rho}$ as above.

(3.0.0) Definition. $\underline{m} \in \mathbf{Z}^{2 n}$ is said to be singular for $X$, $\mathscr{O U}_{\rho}$ (or just for $\rho$ ) in case $\operatorname{det} P_{b}(\underline{m})=0$ for $b=1, \ldots, n$. (Notations as in (2.5.3).) Let $S(\rho)$ denote the subset of $\mathbf{Z}^{2 n}$ consisting of singular indices. An $\underline{m} \notin S(\rho)$ is called nonsingular.

Let $W_{\rho}=\bigoplus W(\chi)$ be the isotypic decomposition. As remarked just after (2.4.0), the $R_{j}$ may be chosen decomposed into blocks according to the isotypic decomposition, and each block in upper triangular form. It follows that $P_{b}(\underline{m})$ is upper triangular, and moreover it is easily seen that the diagonal entry of $P_{b}(\underline{m})$ corresponding to the isotypic $\chi$ is just the $P_{b}(\underline{m})$ of the one-dimensional local system defined by $\chi$. This is because, if $\rho_{j}$ is in upper triangular form, the diagonal entries of 
$R_{j}$ are the logarithms of the diagonal entries of $\rho_{j}$. Thus, the condition det $P_{b}(\underline{m})=0$ becomes the condition "at least one diagonal entry of $P_{b}(\underline{m})$ is 0 ". Or

$$
S(\rho)=\cup S(\chi) \text {. }
$$

(3.0.2) Proposition. Card $S(\rho) \leqslant \operatorname{rank}(\rho)=\operatorname{dim} W_{\rho}$.

Proof. By (3.0.1) this is clearly equivalent to card $S(\chi) \leqslant 1$ for a one-dimensional $\chi$. But $\underline{m} \in S(\chi) \Leftrightarrow \Sigma_{j} \lambda_{j b}\left(2 \pi i m_{j}+R_{j}\right)=0$ for all $b$ where $R_{j}=\log \chi\left(e_{j}\right)$. If $\underline{n} \in S(\chi)$ there is an analogous equation. Subtracting these gives $\Sigma_{j} \lambda_{j b}\left(m_{j}-n_{j}\right)=$ $\overline{0}$. Taking the complex conjugate, $\Sigma \bar{\lambda}_{j b}\left(m_{j}-n_{j}\right)=0$. But this is impossible unless $m_{j}=n_{j}$ for all $j$ because $\operatorname{det}(\lambda, \bar{\lambda}) \neq 0$ by $(1.0 .3)$.

(3.1) Let $W \otimes_{\mathbf{C}} \wedge{ }^{*}\left(\mathbf{C}^{n}\right)$ be the Koszul complex on $T=\left(T_{1}, \ldots, T_{n}\right)$ (cf. (2.6.1)). The following is well known:

(3.1.0) Lemma. If some $T_{j} \in T$ is invertible, the Koszul complex on $T$ is acyclic.

(3.1.1) THEOREM.

$$
\begin{gathered}
g: \mathcal{H}_{\Delta}^{k}\left(\mathscr{W}_{\rho}\right) \tilde{\rightarrow} \mathcal{H}_{\Delta(0)}^{k}\left(\mathscr{W}_{\rho}(0)\right) ; \\
g_{1}: \mathcal{H}_{\square}^{p, q}\left(\mathcal{W}_{\rho}\right) \tilde{\rightarrow} \underset{\underline{m} \in S(\rho)}{\bigoplus} \mathcal{H}_{\square(\underline{m})}^{p, q}\left(\mathcal{W}_{\rho}(\underline{m})\right) .
\end{gathered}
$$

Proof. To see (a) use (2.11.0). We must show $\mathcal{F}_{\Delta(m)}^{k}\left(\mathscr{W}_{\rho}(\underline{m})\right)=0$ if $\underline{m} \neq 0$. But this is the cohomology of the Koszul complex $A^{*}\left(\mathcal{U V}_{\rho}(\underline{m})\right)$ for which $T=$ $\left(A_{1}(\underline{m}), \ldots, A_{2 n}(\underline{m})\right)$ as was remarked in (2.6.1ff). But $\underline{m} \neq 0 \Rightarrow A_{j}(\underline{m})$ is invertible for some $j$ by (2.5.3) and (2.4.0)(d). The result follows now from (3.1.0).

Statement (b) is an immediate consequence of (3.1.0) and the definition of $S(\rho)$.

(3.1.2) COROLlary. The Hodge spectral sequence (2.2.0) admits a decomposition

$$
g: E_{*} \tilde{\rightarrow} \underset{\underline{m} \in S(\rho)}{\bigoplus} E_{*}(\underline{m})
$$

where the abutment

$$
\begin{aligned}
& \text { (a) } \quad \oplus E_{\infty}^{p, q} \stackrel{\simeq}{\rightarrow} \underset{p+q=k}{\oplus} E_{\infty}^{p, q}(0) \simeq \mathcal{H}_{\Delta(0)}^{k}\left(\mathcal{W}_{\rho}(0)\right) \simeq H^{k}\left(X, \underline{W}_{\rho}\right), \\
& \text { (b) } \quad E_{1}^{p, q} \stackrel{\sim}{\rightarrow} \underset{\underline{m} \in S(\rho)}{\bigoplus} E_{1}^{p, q} \simeq \underset{\underline{m} \in S(\rho)}{\bigoplus} \mathcal{H}_{\square(\underline{m})}^{p, q}\left(\mathcal{W}_{\rho}(\underline{m})\right) \simeq H^{q}\left(X, \Omega^{p}\left(\mathcal{W}_{\rho}\right)\right) .
\end{aligned}
$$

The above results give an explicit determination of $H^{k}\left(X, \underline{W}_{\rho}\right)$ and $H^{q}\left(X, \Omega^{p}\left(\mathcal{U}_{\rho}\right)\right)$ in terms of harmonic forms. Everything is reduced to computing in the finitedimensional complexes $A^{*}\left(\mathcal{W}_{\rho}(0)\right)$ and $A^{p, *}\left(\mathscr{W}_{\rho}(\underline{m})\right)$ for $\underline{m} \in S(\rho)$ and determining the finite set $S(\rho)$. This latter will be discussed in the next section.

(3.1.3) Remark. (a) $H^{k}\left(X, \underline{W}_{\rho}\right)=\oplus H^{k}(X, \underline{W}(\chi))$ where $\underline{W}_{\rho}=\oplus \underline{W}(\chi)$ is the isotypic decomposition. I claim $H^{k}(X, \underline{W}(\chi))=(0)$ for all $k$ if $\chi \neq 1$.

Proof. $W(\chi)$ is a successive extension of the one-dimensional local systems defined by the character $\chi$ by itself a certain number of times. Using the long exact cohomology sequence, we are reduced to proving $H^{k}\left(X, \underline{W}_{\chi}\right)=0$ for all $k$ if $\chi \neq 1$ 
and $\operatorname{dim} \chi=1$. But $H^{k}\left(X, \underline{W}_{\chi}\right) \simeq H^{k}\left(A^{*}\left(\mathscr{W}_{\chi}(0)\right)\right)$ by $(3.1 .1)\left(\right.$ a) where $A^{*}\left(\mathscr{W}_{\chi}(0)\right)$ is the Koszul complex on the $\left(A_{1}(0), \ldots, A_{2 n}(0)\right)$. Then, $\chi \neq 1 \Rightarrow$ some $A_{j}(0) \neq 0$ so this complex is acyclic by (3.1.0).

(b) (3.1.2) implies in particular that $H^{k}\left(X, \underline{W}_{\rho}\right) \neq 0$ for some $k \Rightarrow 0 \in S(\rho)$. This can be seen directly as follows: by remark (a), $H^{k}\left(X, \underline{W}_{\rho}\right) \neq 0$ for some $k \Rightarrow$ the trivial character 1 appears in the isotypic decomposition of $\rho$. It is therefore enough, in view of (3.0.1), to show $0 \in S(1)$, which is completely obvious from the definitions.

(c) Phil Griffiths pointed out to me that if $X$ is any compact, Kaehler complex manifold, and $\underline{W}$ is a local system on $X$ whose monodromy representation is unitary, the Hodge spectral sequence of $\underline{W}$ is collapsed at $E_{1}$. One can prove this directly in our case as follows: If $\rho$ is abelian and unitary, $\rho$ is a direct sum of 1-dimensional unitary representations. It is enough to treat the case where $\operatorname{dim} \rho=1, \rho$ unitary. When $\rho=1$ this is just the usual theorem in Kaehler geometry. For $\rho \neq 1$, since by remark (a), $H^{k}\left(X, \underline{W}_{\rho}\right)=0$ for all $k$, we must prove that $H^{q}\left(X, \Omega^{p}\left(\mathcal{Q} \int_{\rho}\right)\right)=0$ for all $(p, q)$. We will do this by showing that $S(\rho)=\varnothing . \underline{m} \in S(\rho) \Leftrightarrow \sum_{j} \lambda_{j b}\left(2 \pi i m_{j}+R_{j}\right)$ $=0$ for all $b$, where $R_{j}=\log \rho\left(e_{j}\right)$. $\rho$ being unitary $\Rightarrow R_{j}$ is pure imaginary. Taking complex conjugate gives $-\Sigma_{j} \bar{\lambda}_{j b}\left(2 \pi i m_{j}+R_{j}\right)=0$ for all $b$. But $\operatorname{det}(\lambda,-\bar{\lambda})=$ $\pm \operatorname{det}(\lambda, \bar{\lambda}) \neq 0$ by (1.0.3), so this implies that $2 \pi i m_{j}+R_{j}=0$ for all $j$, or $R_{j} \in 2 \pi i \mathbf{Z}$ which is impossible because $\rho \neq 1$.

(d) When $\rho$ is semisimple, it may be diagonalized, and hence $A_{j}(\underline{m})$ commutes with $A_{j}(\underline{m})^{*}$ for all $\underline{m}$ and $j$. It follows that the commutator term in (2.12) disappears. Then (3.1.1)(b) may be deduced from the fact that the Hermitian semidefinite matrix $\Sigma_{b} P_{b}(\underline{m})^{*} P_{b}(\underline{m})$ is positive definite if at least one $P_{b}(\underline{m})$ is invertible. The same argument may be made for (3.1.1)(a) if one remarks that $\Delta(\underline{m})$ has exactly the same type of expression as $\square(\underline{m})$ if the differential forms $\varphi$ are expressed in terms of the basis $d v_{I}$ and $P_{b}(\underline{m})$ is replaced by $A_{j}(\underline{m})$.

(e) Warning. If $\rho_{1}$ and $\rho_{2}$ are nonisomorphic representations, the local systems $\underline{W}_{\rho_{1}}$ and $\underline{W}_{\rho_{2}}$ are not isomorphic. It may happen though that

$$
\mathscr{\mathcal { O }} \int_{\rho_{1}}=\mathcal{\theta}_{X}\left(\underline{W}_{\rho_{1}}\right) \tilde{\rightarrow} \mathcal{O}_{X}\left(\underline{W}_{\rho_{2}}\right)=\mathscr{\mathcal { U }} \int_{\rho_{2}} .
$$

This means that a vector bundle may admit inequivalent flat connections.

(3.1.4) REMARK. (a) If $\operatorname{dim} \rho=1$, then $\mathscr{W}_{\rho} \simeq \mathcal{W}_{\rho_{1}}$ where $\rho_{1}$ is the unitary character $\rho /|\rho|$.

(b) Let $E$ be a line bundle on $X$ of chern class 0 ; then the above theorem allows one to deduce the usual results about the cohomology of $\mathcal{L}$ (see [10]).

(c) Let $X$ be an elliptic curve,

$$
\rho(\lambda)=\left(\begin{array}{cc}
1 & \xi(\lambda) \\
0 & 1
\end{array}\right)
$$

where $\xi: L \rightarrow \mathbf{C}$ is an additive map. There is an exact sequence $0 \rightarrow \mathcal{O}_{X}$ $\rightarrow$ Q $\int_{\rho} \rightarrow \mathfrak{E}_{X} \rightarrow 0$ whose extension class is

$$
\xi_{\mathbf{R}}^{\prime} \in \operatorname{Ext}^{1}\left(\mathfrak{C}_{X}, \mathfrak{C}_{X}\right)=H^{1}\left(\mathfrak{C}_{X}\right) \simeq\{f: \mathbf{C} \rightarrow \mathbf{C} \mid f \text { is } \mathbf{C} \text {-antilinear }\}
$$


where $\xi_{\mathbf{R}}=\xi \otimes \mathbf{R}: L \otimes_{Z} \mathbf{R} \rightarrow \mathbf{C}$ and $\xi_{\mathbf{R}}=\xi_{\mathbf{R}}^{\prime}+\xi_{\mathbf{R}}^{\prime \prime}$ where $\xi_{\mathbf{R}}^{\prime}$ (resp. $\xi_{\mathbf{R}}^{\prime \prime}$ ) is $\mathbf{C}$-antilinear (resp. C-linear) (see [10, Chapter I]). If $\rho_{1}$ and $\rho_{2}$ are such that the sequence does not split, one can show that $\mathcal{W}_{\rho_{1}} \simeq \mathcal{W}_{\rho_{2}}$ and, moreover,

$$
\operatorname{dim} H^{\circ}\left(X, \mathscr{\vartheta}_{\rho}\right)=\operatorname{dim} H^{1}\left(X, \mathscr{W}_{\rho}\right)=1
$$

if $\rho=\rho_{1}$ or $\rho_{2}$. These results are contained in those of Atiyah [0].

\section{A consequence of Baker's theory.}

(4.0) Let $\overline{\mathbf{Q}}$ be the algebraic closure of $\mathbf{Q}$ in $\mathbf{C}$. Alan Baker [1] has proved (a more precise version of) the following

(4.0.0) Theorem. Let $\alpha_{1}, \ldots, \alpha_{n} \in \overline{\mathbf{Q}}^{*}$. Let $\xi_{1}, \ldots, \xi_{n} \in \mathbf{C}$ be such that $\exp \left(\xi_{j}\right)=\alpha_{j}$. If the $\xi_{j}$ are linearly independent over $\mathbf{Q}$, then they are linearly independent over $\mathbf{Q}$.

(4.0.1) CoRollary (CF. [11]). Let $\lambda_{1}, \ldots, \lambda_{n} \in \overline{\mathbf{Q}}$ be Q-linearly independent, $\alpha_{1}, \ldots, \alpha_{n} \in \overline{\mathbf{Q}}^{*}$ and $\xi_{1}, \ldots, \xi_{n} \in \mathbf{C}$ with $\exp \xi_{j}=\alpha_{j}$. If at least one $\xi_{j} \neq 0$, then $\Sigma_{j} \lambda_{j} \xi_{j} \neq 0$.

Proof. Assume $\xi_{1} \neq 0$. If $n=1$, the proposition is trivial. We do an induction on $n$. Assume that the proposition is true for $n$ but false for $n+1$. We then have an equation $\lambda_{1} \xi_{1}+\cdots+\lambda_{n+1} \xi_{n+1}=0$. But then by (4.0.0) we can find a relation $\mu_{1} \xi_{1}+\cdots+\mu_{n+1} \xi_{n+1}=0$ where all $\mu_{j} \in \mathbf{Q}$, but not all $\mu_{j}=0$. In fact, there is a $\mu_{k} \neq 0$ with $k>1$ because $\mu_{1} \neq 0$ and $\mu_{j}=0$ for all $j>1$ is impossible. Let this $k=2$. Multiply the first equation by $\mu_{2}$ and the second equation by $\lambda_{2}$ and subtract to get

$$
\left(\mu_{2} \lambda_{1}-\mu_{1} \lambda_{2}\right) \xi_{1}+\left(\mu_{2} \lambda_{3}-\mu_{3} \lambda_{2}\right) \xi_{3}+\cdots+\left(\mu_{2} \lambda_{n+1}-\mu_{n+1} \lambda_{2}\right) \xi_{n+1}=0 .
$$

But it is easy to see that the set $\left\{\left(\mu_{2} \lambda_{j}-\mu_{j} \lambda_{2}\right) \mid j=1,3, \ldots, n+1\right\}$ is Q-linearly independent, so the above equation contradicts the induction hypothesis.

(4.0.2) ReMARK. If you assume moreover that $1, \lambda_{1}, \ldots, \lambda_{n}$ are $\mathbf{Q}$-linearly independent, you can prove in exactly the same way that $\alpha_{1}^{\lambda_{1}} \cdots \alpha_{n}^{\lambda_{n}}$ is a transcendental number.

(4.0.3) Proposition. Let $\mho_{\rho}$ be a flat vector bundle on a complex torus $X$ whose monodromy representation $\rho$ is isotypic for a character $\chi$ such that $\chi(\lambda) \in \overline{\mathbf{Q}}^{*}$ for all $\lambda \in L$ and that at least one of the sets $\left(\lambda_{1 k}, \ldots, \lambda_{2 n, k}\right)((1.0 .1))$ is $\overline{\mathbf{Q}}$-valued and Q-linearly independent. Then, if $\chi \neq 1, H^{q}\left(X, \Omega^{p}\left(\mathcal{\vartheta}_{\rho}\right)\right)=0$ for all $(p, q)$.

Proof. We will prove that $S(\rho)=S(\chi)$ is empty and apply ((3.1.2)) $\underline{m} \in S(\chi) \Leftrightarrow$ $\Sigma_{j} \lambda_{j b}\left(2 \pi i m_{j}+\log \chi_{j}\right)=0$ for all $b$. Let $\xi_{j}=2 \pi i m_{j}+\log \chi_{j}$. Then $\exp \left(\xi_{j}\right)=\chi_{j}=$ $\chi\left(e_{j}\right) \in \overline{\mathbf{Q}}^{*}$. If $\chi \neq 1$, at least one $\xi_{j} \neq 0$. Hence the result follows from (4.0.1).

(4.1) Suppose now that $X$ is an elliptic curve $(\operatorname{dim} X=1)$. We can assume that the lattice $L$ is generated by $1, z=z_{1}+i z_{2}$ with $z_{2}>0$. By (1.0.1) it is easy to see that $2 \lambda_{1}=1-i z_{1} / z_{2}, 2 \lambda_{2}=i / z_{2}$. If $X$ has a complex multiplication (is of CM type) then $z$ is an imaginary quadratic number, so that $z_{1} \in \mathbf{Q}$ and $z_{2} \in \overline{\mathbf{Q}}$ but $z_{2} \notin \mathbf{Q}$. 
Then it is easy to show that $\lambda_{1}, \lambda_{2} \in \overline{\mathbf{Q}}$ are $\mathbf{Q}$-linearly independent. It follows immediately from (4.0.3) that

(4.1.0) Proposition. Let $X$ be an elliptic curve of CM type, $\mathcal{Q f}_{\rho}$ a flat vector bundle on $X$ whose monodromy representation $\rho$ is isotypic for a character $\chi$ which is $\overline{\mathbf{Q}}^{*}$-valued and nontrivial. Then $H^{q}\left(X, \Omega^{p}\left(\mathcal{W}_{\rho}\right)\right)=0$ for all $(p, q)$.

The result is also true for any $\mathcal{W}_{\rho}$ which is a direct sum of vector bundles of that form.

\section{A geometric application.}

(5.0) Let $f: X \rightarrow S$ be a proper smooth morphism of complex manifolds. The local system $R^{i} f_{*} \mathbf{C}$ is the sheaf of horizontal sections in $\mathbf{R}^{i} f_{*}\left(\Omega_{X / S}^{*}\right)$ for the Gauss-Manin connection $\nabla$. Here, $\Omega_{X / S}^{*}$ denotes the relative de Rham complex and $\mathbf{R} f_{*}$ denotes the hyperderived functor. If $f$ is algebrizable then so is the Gauss-Manin connection. The monodromy representation $\rho$ for this situation preserves the $\mathbf{Q}$-local system $R^{i} f_{*} \mathbf{Q}$ and hence $\rho$ is equivalent to a representation in $\operatorname{GL}(\mathbf{Q})$. In particular, the eigenvalues of this representation are all $\overline{\mathbf{Q}}^{*}$-valued. Hence

(5.0.0) LeMma. $f: X \rightarrow S$ a proper smooth morphism of complex manifolds where $S$ is a complex torus. Then the diagonal characters occurring in $R^{i} f_{*} \mathbf{C}$ are $\overline{\mathbf{Q}}^{*}$-valued.

(5.1) Let $f: X \rightarrow S$ be an analytically locally trivial map where $S$ is an elliptic curve and $X$ is a smooth compact threefold such that the fiber of $f$ is a 2-dimensional complex torus $A$. These are 3-dimensional analogues of hyperelliptic surfaces [12]. We have $R^{j} f_{*} \mathrm{C}=\wedge^{j} R^{1} f_{*} \mathrm{C}$. Note that the monodromy representation $\rho$ is induced by an analytic automorphism of fiber $A$. Such an automorphism must preserve the Hodge decomposition of the fiber. That is:

(5.1.0) LEMMA.

$$
R^{j} f_{*} \mathbf{C} \simeq \underset{p+q=j}{\bigoplus} \underline{H}^{p, q} \bar{H}^{p, q}=\underline{H}^{q, p}
$$

where $\underline{H}^{p, q}$ is the sublocal system corresponding to the $(p, q)$ part of the Hodge decomposition of the fiber of $f$.

We can also write

$$
\rho^{j}=\bigoplus_{p+q=j} \rho^{p, q}, \overline{\rho^{p, q}}=\rho^{q, p} .
$$

In particular, $R^{1} f_{*} \mathbf{C}=\underline{H}^{1,0} \oplus \underline{H}^{0,1}$. Let $\chi_{1}, \chi_{2}$ be the diagonal characters of $\rho^{1,0}$. Then $\bar{\chi}_{1}, \bar{\chi}_{2}$ are the diagonal characters of $\rho^{0,1}$.

(5.2) We have the Leray spectral sequence

$$
E_{2}^{i j}=H^{i}\left(S, R^{i} f_{*} \mathbf{C}\right) \Rightarrow H^{i+j}(X, \mathbf{C})
$$

and its sequence of low degree

$$
0 \rightarrow H^{1}\left(S, f_{*} \mathbf{C}\right) \rightarrow H^{1}(X, \mathbf{C}) \rightarrow H^{0}\left(S, R^{1} f_{*} \mathbf{C}\right) \rightarrow H^{2}\left(S, f_{*} \mathbf{C}\right) \rightarrow H^{2}(X, \mathbf{C}) .
$$


We see from this and $f_{*} \mathbf{C}=\mathbf{C}$ that $b_{1}(X)=\operatorname{dim} H^{1}(X, \mathbf{C}) \geqslant 2$. But, if $b_{1}(X)=2$, then $H^{0}\left(S, R^{1} f_{*} \mathrm{C}\right)$ injects into $H^{2}\left(S, f_{*} \mathrm{C}\right)$ which is 1-dimensional. So $b_{1}(X)=2 \Rightarrow$ $h^{0}\left(R^{1} f_{*} \mathrm{C}\right) \leqslant 1$. But, if $h^{0}\left(R^{1} f_{*} \mathrm{C}\right)=h^{0}\left(\underline{H}^{1,0}\right)+h^{0}\left(\underline{H}^{0,1}\right)>0$ then at least one of the diagonal characters, say $\chi_{1}$, is trivial because of (3.1.3)(a). It follows then that both $\underline{H}^{1,0}$ and $\underline{H}^{0,1}$ contain the trivial character as an isotypic component. But any local system on a complex torus which is isotypic for a character $\chi$ is a successive extension of the one-dimensional local system defined by $\chi$. Hence, if a local system contains the trivial character, the long exact cohomology sequence shows that it must have at least one global section. We conclude from this that $h^{0}\left(\underline{H}^{1,0}\right) \geqslant 1$ and $h^{0}\left(H^{0,1}\right) \geqslant 1$, or $h^{0}\left(R^{1} f_{*} \mathbf{C}\right)>0 \Rightarrow h^{0}\left(R^{1} f_{*} \mathbf{C}\right) \geqslant 2$. Or

$$
b_{1}(X)=2 \Leftrightarrow h^{0}\left(R^{1} f_{*} \mathbf{C}\right)=0 \quad\left(\Leftrightarrow \chi_{1}, \chi_{2} \neq 1\right) .
$$

(5.2.1) Lemma. Situation as above: (a) $f_{*} \theta_{X}=\theta_{S}$; (b) $R^{1} f_{*} \theta_{X}=\underline{H}^{0,1} \otimes_{C} \theta_{S}$; (c) $\Omega_{X / S}^{1}=f^{*}\left(\underline{H}^{1,0} \otimes_{\mathbf{C}} \theta_{S}\right)$.

Proof. (a) Immediate from Stein factorization. (b) Clear from definitions. To see (c), $f$ is locally over $S$ isomorphic to the second projection $A \times \Delta \rightarrow \Delta, \Delta \subset S$ a small disk. Because the cotangent bundle of $A$ is trivial, it is clear that $\Omega_{X / S}^{1}$ when restricted to $A \times \Delta$ is isomorphic with $H^{0}\left(A, \Omega_{A}^{1}\right) \otimes_{\mathbf{C}} \theta_{X}=f^{*}\left(\underline{H}^{1,0} \otimes_{\mathbf{C}} \vartheta_{S}\right)$.

(5.2.2) Proposition. Let $X$ be a compact, complex threefold, $f: X \rightarrow S$ an analytically locally trivial fibering where $S$ is a CM elliptic curve and the fiber of $f$ is a 2-dimensional complex torus. Then $b_{1}(X)=2 \Rightarrow h^{0}\left(\Omega_{X}^{1}\right)=h^{1}\left(\theta_{X}\right)=1$.

Proof. The Leray spectral sequence $E_{2}^{p, q}=H^{p}\left(S, R^{q} f_{*} \theta_{X}\right) \Rightarrow H^{p+q}\left(X, \theta_{X}\right)$ gives 2 composition factors $E_{2}^{1,0}$ and $E_{2}^{0,1}$ to $H^{1}\left(X, \theta_{X}\right)$. Since $\operatorname{dim} S=1, E_{2}^{0,1}=E_{\infty}^{0,1}$ and similarly for $E_{2}^{1,0}$. To show $h^{1}\left(\theta_{X}\right)=1$, it is enough to prove $\operatorname{dim}\left(E_{2}^{1,0} \oplus E_{2}^{0,1}\right)=1$.

Now $E_{2}^{1,0}=H^{1}\left(S, f_{*} \theta_{X}\right)=H^{1}\left(S, \theta_{S}\right)$ by (5.2.1). It is one dimensional. We must prove $E_{2}^{0,1}=0$. But $E_{2}^{0,1}=H^{0}\left(S, R^{1} f_{*} \theta_{X}\right)=H^{0}\left(S, \underline{H}^{0,1} \otimes_{\mathrm{C}} \theta_{S}\right)$ by (5.2.1). But (5.2.0) tells us that the diagonal characters of $\underline{H}^{0,1}$ are nontrivial. As they are also $\overline{\mathbf{Q}}^{*}$-valued and $S$ is of CM type, the result follows from (4.1.0)

As to $h^{0}\left(\Omega_{X}^{1}\right)$, we have an exact sequence $0 \rightarrow f^{*} \Omega_{S}^{1} \rightarrow \Omega_{X}^{1} \rightarrow \Omega_{X / S}^{1} \rightarrow 0$ which gives $0 \rightarrow H^{0}\left(f^{*} \Omega_{S}^{1}\right) \rightarrow H^{0}\left(\Omega_{X}^{1}\right) \rightarrow H^{0}\left(\Omega_{X / S}^{1}\right) \rightarrow \cdots$. Now, $H^{0}\left(X, f^{*} \Omega_{S}^{1}\right)=$ $H^{0}\left(S, f_{*} f^{*} \Omega_{S}^{1}\right)$ by Leray. But

$$
f_{*} f^{*} \Omega_{S}^{1}=f_{*}\left(f^{*} \Omega_{S}^{1} \otimes_{\Theta_{X}} \theta_{X}\right) \simeq \Omega_{S}^{1} \otimes_{\Theta_{S}} f_{*} \vartheta_{X} \simeq \Omega_{S}^{1}
$$

by the projection formula and (5.2.1). So $H^{0}\left(f_{*} \Omega_{S}^{1}\right)$ is one dimensional, and it is enough to prove $H^{0}\left(\Omega_{X / S}^{1}\right)=0$. Using Leray again, $H^{0}\left(X, \Omega_{X / S}^{1}\right) \simeq H^{0}\left(S, f_{*} \Omega_{X / S}^{1}\right)$. By (5.2.1) and the projection formula,

$$
f_{*} f^{*} \Omega_{X / S}^{1}=f_{*}\left(f^{*}\left(\underline{H}^{1,0} \otimes_{\mathbf{C}} \theta_{S}\right)\right) \simeq\left(\underline{H}^{1,0} \otimes_{\mathbf{C}} \theta_{S}\right) \otimes_{\vartheta_{S}} f_{*} \theta_{X} \simeq \underline{H}^{1,0} \otimes_{\mathbf{C}} \theta_{S} .
$$

As before, $\underline{H}^{1,0}$ is a local system whose diagonal characters are $\overline{\mathbf{Q}}^{*}$-valued and nontrivial so the result follows from (4.1.0)

(5.2.3) Remark. $X$ is a locally trivial fibering of a Kaehler manifold by a Kaehler manifold. But $X$ need not be Kaehler (in which case (5.2.2) is evident). We will see in a moment that the condition that $S$ be of CM type cannot be relaxed in (5.2.2). 
(5.2.4) Counterexample. Let

$$
K=\mathbf{Q}(\sqrt{2}, i)=\mathbf{Q}(\zeta), \quad \zeta=e^{2 \pi i / 8}=\sqrt{2} / 2+i \sqrt{2} / 2,
$$

be an imaginary quadratic extension of the totally real field $\mathbf{Q}(\sqrt{2})$. Let $\mathfrak{o}=\mathbf{Z}[\sqrt{2}, i]$ a (nonmaximal) order in $K$. We have a canonical isomorphism $K \otimes_{\mathbf{Q}} \mathbf{R} \tilde{\rightarrow} \mathbf{C}^{2}$ which comes from the two nonconjugate archimedean valuations of $K$. If $\sigma \in \mathrm{Gal}(K / \mathbf{Q}(i))$ is the generator, the canonical map $\mathfrak{o} \rightarrow K \otimes_{\mathbf{Q}} \mathbf{R} \tilde{\rightarrow} \mathbf{C}^{2}$ is given by

$$
\begin{aligned}
\alpha & =a+b \sqrt{2}+c i+d i \sqrt{2} \rightarrow\left(\begin{array}{c}
\alpha \\
\boldsymbol{\alpha}^{\sigma}
\end{array}\right) \\
& =\left(\begin{array}{l}
a+b \sqrt{2}+c i+d i \sqrt{2} \\
a-b \sqrt{2}+c i-d i \sqrt{2}
\end{array}\right)
\end{aligned}
$$

and imbeds $\mathrm{o}$ as a lattice in $\mathbf{C}^{2}$. Let $A=\mathbf{C}^{2} / \mathrm{o}$.

Let $\varepsilon=-1+\sqrt{2}$, which is a unit in $\mathfrak{o} .\left(z_{1}, z_{2}\right) \rightarrow\left(\varepsilon z_{1}, \varepsilon^{\sigma} z_{2}\right)$ is an automorphism of $\mathbf{C}^{2}$ inducing an automorphism of $\mathfrak{o}$, so it gives an automorphism of $A=\mathbf{C}^{2} / \mathfrak{o}$. Let $L=\mathbf{Z} e_{1} \oplus \mathbf{Z} e_{2} \subset \mathbf{C}$, some lattice to be specified shortly. $S=\mathbf{C} / L$. We have a diagram

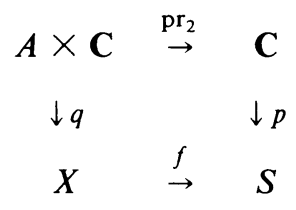

$p$ is the natural projection, $q$ is the quotient map for the action of $L$ on $A \times \mathbf{C}$ given by

$$
\left(\left(z_{1}, z_{2}\right), w\right) \rightarrow\left(\left(\varepsilon^{\mu_{1}} z_{1},\left(\varepsilon^{\sigma}\right)^{\mu_{1}} z_{2}\right), w+\mu\right)
$$

for $\mu=\mu_{1} e_{1}+\mu_{2} e_{2} \in L$. Let $\chi_{1}(\mu)=\varepsilon^{\mu_{1}}, \chi_{2}(\mu)=\left(\varepsilon^{\sigma}\right)^{\mu_{1}}$. Then clearly, $\rho^{1,0}=\chi_{1} \oplus$ $\chi_{2}=\rho^{0,1}$. Since the identity character does not appear in $\rho^{1,0} \oplus \rho^{0,1}$, we have $h^{0}\left(R^{\mathrm{l}} f_{*} \mathrm{C}\right)=0$ so $b_{1}(X)=2$ by $(5.2 .0)$.

Now, we will find an $L$ such that $h^{0}\left(\underline{H}^{0,1} \otimes_{\mathbf{C}} \theta_{S}\right) \geqslant 1$. By the argument in (5.2.2) this gives $h^{1}\left(\theta_{X}\right) \geqslant 2$. Because $\underline{H}^{0,1}$ is the direct sum of two 1-dimensional local systems, to get a holomorphic section, we need only insure that one of the characters $\chi_{1}$ or $\chi_{2}$ has a singular index relative to $L$. (If a one-dimensional local system has a singular index, then it has a holomorphic section. This is easily proved from the definitions.) We must find an $L$ so that $\lambda_{1}\left(2 \pi i m_{1}+\log \varepsilon\right)+\lambda_{2}\left(2 \pi i m_{2}\right)=0$ for some $m_{1}, m_{2} \in \mathbf{Z}$. Set $\lambda_{1}=-2 \pi i, \lambda_{2}=\log \varepsilon, m_{2}=1, m_{1}=0$. Note that $\lambda_{1}, \lambda_{2}$ correspond to a lattice under (1.0.2) because

$$
\operatorname{det}\left(\frac{\lambda_{1}}{\lambda_{1}} \frac{\lambda_{2}}{\lambda_{2}}\right) \neq 0
$$

The crucial point here is that the monodromy is not unitary. For in that case, $\log \varepsilon$ would be pure imaginary and the above determinant would $=0$.

(5.2.5) Questions. (Hironaka) (a) Is there a Kaehler manifold among the $X$ in (5.2.2)? If so, the above counterexample might provide another example of a 
non-Kaehlerian deformation of a Kaehler manifold. One knows that such examples exist, but the ingenious construction in [8] is quite complicated.

(b) Are there algebraic threefolds $X$ which admit a fibering $f: X \rightarrow S$ of the type discussed above with infinite monodromy? This never happens in the case of hyperelliptic surfaces for a trivial reason. (The automorphism group of any elliptic curve is finite.)

(5.3) Remarks. (a) Deligne has proved [4] a degeneration theorem which asserts that if $X$ is Kaehler, or if $f: X \rightarrow S$ is a proper smooth morphism of smooth schemes, the Leray spectral sequence (5.2) over $\mathbf{Q}$ degenerates. This has (5.2.0) as an immediate consequence.

(b) If $X$ is algebraic, and $f$ is a proper, smooth morphism of schemes, Deligne has proved [3] that the monodromy representation is semisimple. This means that $\rho$ decomposes as a direct sum of one-dimensional representations, and the main theorems of this paper may be deduced from the one-dimensional case in that case.

\section{REFERENCES}

0. M. F. Atiyah, Vector bundles on an elliptic curve, Proc. London Math. Soc. 7 (1957), 414-452.

1. A. Baker, Transcendental number theory, Cambridge Univ. Press, New York, 1975.

2. P. Deligne, Équations différentielles à points, Singuliers Réguliers, Lecture Notes in Math., vol. 163, Springer-Verlag, Berlin and New York.

3.

4. __ Théorème de Lefschetz et critères de dégénérescence de suites spectrales, Inst. Hautes Études Sci. Publ. Math. 35 (1968), 107-126.

5. P. Griffiths and W. Schmid, Recent developments in Hodge theory, Proc. Internat. Colloq. on Discrete Subgroups of Lie Groups and Applications to Moduli, Oxford Univ. Press, published for the Tata Institute, Bombay, 1975, pp. 31-127.

6. A. Grothendieck, Sur quelques points d' algèbre homologique, Tôhoku Math. J. 9 (1957), 119-221.

7. __ Sur le mémoire de Weil: Généralisation des fonctions Abéliennes, Séminaire Bourbaki, Exposé $141,1956 / 57$.

8. H. Hironaka, An example of a non-Kaehlerian complex analytic deformation of Kaehlerian complex structures, Ann. of Math. (2) 75 (1962), 190-208.

9. J. Morrow and K. Kodaira, Complex manifolds, Holt, Rinehart, Winston, New York, 1971.

10. D. Mumford, Abelian varieties, Oxford Univ. Press, New York, 1970.

11. J. P. Serre, Travaux de Baker, Séminaire Bourbaki, Exposé 368, 1969/70.

12. T. Suwa, On hyperelliptic surfaces, J. Math. Soc. Japan. 22 (1970), 469-476.

Department of Mathematics, Louisiana State University, Baton Rouge, Louisiana 70803 